\title{
Neuromuscular Contacts Induce Nitric Oxide Signals in Skeletal Myotubes in vitro
}

\author{
Britta Püttmann ${ }^{a}$ Eva-Maria Gerlach ${ }^{b}$ Mark Krüger ${ }^{a}$ Dieter Blottner ${ }^{a}$ \\ aNeurobiology and Neuromuscular Working Group, Institute of Anatomy, Campus Benjamin Franklin, \\ Charité-University Medicine Berlin, and bMax Planck Institute for Infection Biology, Campus Charité, \\ Berlin, Germany
}

\section{Key Words}

Agrin - Co-cultures · In vivo labeling · Motoneuron .

Neuromuscular synapses $\cdot$ Nitric oxide $\cdot$ Skeletal

muscles

\begin{abstract}
It has previously been shown that skeletal myotubes express nitric oxide synthase (NOS) and produce and release NO signals. NOS is also part of agrin-induced acetylcholine receptor aggregations on myotubes. As nerve-muscle interactions underlie reciprocal signaling mechanisms, we hypothesized that NO signals in target myotubes may be induced by neuromuscular contacts in development. Chimeric neuron-myotube co-cultures were prepared using p75-selected spinal cord neurons from embryonic chicken. Confocal imaging revealed robust 1,2-diaminoanthraquinone red fluorescence indicative of de novo formation of NO only in those myotubes which were contacted by neurites, also verified by preand postsynaptic marker costaining (anti-synaptotagmin and $\alpha$-bungarotoxin). Neither soluble agrin nor sensory dorsal root ganglionic neurons showed comparable effects in this model. We concluded that in target skeletal muscle cells the NOS/NO system is controlled by moto-
\end{abstract}

neuron contacts by as yet incompletely understood signaling mechanisms. Endogenous NO signaling in myotubes may be essential during synapse formation and plasticity of the neuromuscular system.

Copyright $\odot 2005$ S. Karger AG, Basel

\section{Introduction}

Nitric oxide (NO) signals are fundamental in the development and function of the central and peripheral nervous system [1]. The dimeric and gaseous NO molecules produced by NO synthases (NOS) act as freely diffusible messengers in defined neurons in the central and peripheral nervous system [2]. NO addresses cellular and subcellular targets in defined neuronal networks [3, 4], e.g. nitrosylation [5]. The NOS gene has a complex structure [6] and three major isoforms of NOS, neuronal (NOS-1), macrophage (NOS-2) and endothelial (NOS-3), have been cloned and characterized [7]. NOS is abundantly expressed in peripherally innervated organs suggesting important functions of target-derived NO signals [8].

While somatic motoneurons in the spinal cord normally lack NOS expression [9] NOS is abundantly found in their target skeletal muscle fibers representing a major

\section{KARGER}

Fax +41613061234 E-Mail karger@karger.ch www. karger.com (c) 2005 S. Karger AG, Base

1424-862X/05/0143-0085\$22.00/0

Accessible online at:

www. karger.com/nsg
Prof. Dr. rer. nat. Dieter Blottner, AG Neurobiologie und Neuromuskuläres System Institut für Vegetative Anatomie und Zentrum für Weltraummedizin Berlin Campus Benjamin Franklin, Charité-University Medicine Berlin, Arnimallee 22 DE-14195 Berlin (Germany)

Tel. +49308445 1629, Fax +49308445 1658, E-Mail dieter.blottner@charite.de 
physiological source of NO in the body [10-13]. In the muscle fiber, $\mathrm{NO}$ is generated by $\mathrm{Ca}^{2+}$-dependent constitutively expressed NOS-1 or by one of its coexpressed muscle-specific splice variants termed NOS- $\mu$ [14]; both are associated with the sarcolemmal dystrophin-associated glycoprotein complex (DAGC) of normal adult muscle fibers [15]. NOS-1 is concentrated at the neuromuscular junction [16-18] together with cyclic GMP-dependent protein kinases and soluble guanylyl cyclase $[19,20]$ and is part of agrin-induced nicotinic acetylcholine receptor (AChR) aggregations on myotubes including NMDAreceptor, ion channels, PSD-95 or 43K-rapsyn linker proteins, suggesting fundamental signaling mechanisms of NO in neuromuscular synapse formation [21-24].

Skeletal mouse C2C12 myotubes express a concomitant cytosolic pattern of NOS-1-NOS-3 isoforms in culture [25]. During postnatal development, NOS becomes redistributed during functional skeletal muscle innervation [26]. Endogenous NO is a retrograde signal involved in nerve terminal differentiation and neuromuscular junction plasticity [27, 28] and development [29]. Whether endogenous formation of NO molecules in target skeletal myotubes is controlled by neurons is presently unknown. We, therefore, prepared chimeric neuron/myotube co-cultures in order to determine whether NO formation in skeletal myotubes is induced by neuronal contacts. Viable C2C12 myotubes were preloaded with 1,2-diaminoanthraquinone (DAQ), a red-fluorescent NO-specific probe for intracellular detection. The DAQ-preloaded myotubes were co-cultured with embryonic chicken p75selected spinal cord motoneurons or sensory dorsal root ganglionic (DRG) neurons. In p75 neuron/myotube cocultures, robust DAQ red fluorescence staining was detectable in the sarcosol of target myotubes by confocal laser microscopy thus indicating intracellular de novo formation of NO. Soluble agrin or sensory neurons failed to show similar effects in this model. Our in vitro experiments confirmed that endogenous NO synthesis in target myotubes might be under the control of neuromuscular contacts of somatic motoneurons.

\section{Materials and Methods}

Mouse skeletal C2C12 myoblasts (ATTC, Rockville, Md., USA) were cultured in proliferation medium [DMEM with $4.5 \mathrm{~g} / 1$ glucose/ $1 \%$ non-essential amino acids (Biochrom, Berlin, Germany) $/ 1 \%$ penicillin-streptomycin, P/S (Gibco, Karlsruhe, Germany)/1\% L-glutamine (Biochrom) $/ 10 \%$ fetal calf serum (FCS; PAA Laboratories, Marburg, Germany) under $6 \% \mathrm{CO}_{2}$ atmosphere in a $37^{\circ} \mathrm{C}$ incubator (humidity 95\%)]. Myotube formation was induced within 2-3 days in vitro following exchange of the fusion medium (DMEM $1 \mathrm{~g} / \mathrm{l} \mathrm{glu-}$ cose $/ 1 \% L$-glutamine $/ 1 \% \mathrm{P} / \mathrm{S} / 2 \% \mathrm{FCS}$ ), as previously described [23].

Five- to 6-day-old chick embryos (E5/E6) were obtained from fertilized white leghorn eggs and dissected under sterile conditions. Embryonic trunks were dissected at the ventral midline, the thoracolumbar spinal cord enlargements Th 12 to L 5 were removed following laminectomy and discretely microdissected into ventral horn tissue columns incubated in ice-cold Hank's salt solution (HSS, Gibco), washed twice with ice-cold HSS, and incubated in $0.025 \%$ trypsin/ HSS $\left(37^{\circ} \mathrm{C}\right.$ water bath for $\left.30 \mathrm{~min}\right)$. The tissue pieces were dissociated and triturated in $1 \mathrm{ml}$ of DMEM/2\% FCS/DNase I $(0.5 \mathrm{mg} /$ $\mathrm{ml}$, Boehringer, Mannheim, Germany) by siliconized glass pipettes. In addition, lumbar (L1-L5) DRG neurons were isolated from E8 chicken embryos. Chick embryonic neurons were purified by preplating steps as previously described [30].

A rabbit anti-mouse antibody which recognizes the extracellular domain of the p 75 low-affinity nerve growth factor receptor (NGFR) expressed by the pool of spinal motoneuron cells at developmental stages E5/E6 [31] and which is cross-reactive with the chicken p75 NGFR (Chemicon, Hofheim, Germany) was used for 'cell panning': 35-mm culture dishes (Nunc, Roskilde, Denmark) were first coated with a rabbit-IgG Fc-specific antibody (Dianova, Hamburg, Germany) dissolved in PBS (1:150) for $2 \mathrm{~h}$ at room temperature (RT). Following three washes in PBS, the dishes were incubated with rabbit anti-mouse p75 NGFR antibody, diluted 1:100 in PBS for $2 \mathrm{~h}$ at RT. After three washing steps with DMEM/2\% FCS, $2 \mathrm{ml}$ of the preplated neuron-like cells were seeded into p75 NGFR-precoated 35-mm dishes (seeding rate: 200,000 cells/dish) and incubated for $30 \mathrm{~min}$ at RT. The neuronal cells captured by immobilized $\mathrm{p} 75$ NGFR antibodies at the bottom of the dish were mechanically remobilized and harvested in $2 \mathrm{ml}$ of DMEM $/ 2 \% \mathrm{FCS}$, centrifuged ( $300 \mathrm{~g}$, $2 \mathrm{~min}$ ) and recounted. Cell panning yielded about 250,000 living cells $/ \mathrm{ml}$ (Trypan blue exclusion test), about $92.2 \pm 1.2 \%(\mathrm{n}=6$, two independent experiments) of these cells were immunopositive for the E5/E6 chick motoneuron-specific marker calcitonin-gene related peptide (anti-CGRP monoclonal antibody, 1:2,500 in PBS, Amersham, Amersham, UK) as described [32].

Purified neurons were seeded onto fused $\mathrm{C} 2 \mathrm{C} 12$ myotube monolayers grown onto uncoated glass coverslips (seeding rate: 1,000 neurons/13-mm-diameter coverslip) and cocultivated in DMEM/2\% FCS supplemented with $2 \%$ chicken embryo extract (Gibco) and raised as chick-mouse chimeric co-cultures for up to 8 days in vitro. Neurites were immunostained with anti-neurofilament (NF) H polyclonal antibodies (Chemicon), diluted 1:500 in PBS, for $2 \mathrm{~h}$ at RT followed by secondary Cy2-conjugated anti-rb antibody (Dianova) diluted 1:100 for $1 \mathrm{~h}$ at RT. As presynaptic marker, we used a monoclonal antibody directed against the $65-\mathrm{kDa}$ band of the synaptic vesicle protein synaptotagmin, which is identical in humans, rats and chicken (clone ASV 48, StressGen, Victoria, Canada), diluted 1:200 in PBS, for $2 \mathrm{~h}$ at RT followed by anti-mouse Cy2 (1:100, $1 \mathrm{~h}$ at RT). As postsynaptic markers, we used Texas-red-conjugated $\alpha$-bungarotoxin $\left(\alpha \mathrm{BGTx}\right.$, Molecular Probes, Eugene, Oreg.; $10^{-8} M, 2-4 \mathrm{~h}$ at $37^{\circ} \mathrm{C}$ ) or, green-fluorescent Alexa Fluor 488 -conjugated $\alpha \mathrm{BGTx}$, both specifically bind to the $\beta$-subunit of the nicotinic AChR on the myotube membrane [23]. As controls, aneural myotubes, i.e. myotube cultures without neurons, were subjected to identical staining protocols $(\mathrm{n}=3)$. As further control, the first antibody was omitted from the staining protocol. Quantifications were made by counting the numbers of BGTx fluorescence-labeled AChR clusters per 
$100 \mu \mathrm{m}$ myotube segments $(\mathrm{n}=40)$ in (i) motoneuron-myotube cocultures, (ii) aneural myotubes and (iii) myotube cultures supplemented overnight with chick motoneuron conditioned medium. Values are given as means \pm SEM.

NO formation was measured in supernatant culture medium using a quantitative colorimetric NO detection kit (Cayman Chemical, Ann Arbor, Mich., USA) based on the photometric nitrate/nitrite assay with the Griess reagent (detection limit $2 \mu M$ ) in 96-well titer plates (Nunc) according to the manufacturer's protocol. NO formation was inhibited in fused myotubes cultured for 2-3 days in vitro ( $\mathrm{n}=12$ ) by 1 -hour treatment with $1 \mu M$ of diphenyleiodonium chloride, n $\omega$-nitro- $L$-arginine, both non-specific NOS blockers [32], or by $100 \mathrm{n} M$ of 3-bromo-7-nitroindazole and 2-amino-5,6, dihydro-6methyl-4H-1,3-thiazine $\mathrm{HCl}$ (AMT), both semi-selective blockers for NOS-1 and NOS-2 (Alexis, Grünberg, Germany; [34]). Following treatments with the inhibitors listed, all 96-well plates were directly assayed at $540 \mathrm{~nm}$ wavelength (Philips PV8625 UV/VIS) in order to avoid false-positive NO reaction signals due to extended reaction time. The nitrite/nitrate concentrations were calculated as nitrite + nitrate $(\mu M)=(\mathrm{NO})_{\text {medium }}$ and given as micromolar $(\mu M)$ concentrations (means \pm SEM). Nitrite/nitrate linear standard curves were determined by triple determinations ( $\mathrm{n}=3$, not shown).

Direct visualization of intracellular NO production in viable myotubes was accomplished by DAQ (Molecular Probes) which reacts with $\mathrm{NO}$ to form a red fluorescent and stable precipitate that accumulates in viable myotubes [35] or neuronal cells [36]. DAQ was diluted from a stock solution kept in darkness (1 g/ml DMSO) to 1:50 in prewarmed DMEM. Fused myotubes were preloaded with DAQ for $30-40 \mathrm{~min}$ at $37^{\circ} \mathrm{C}$ in $5 \% \mathrm{CO}_{2}$ atmosphere. Excess DAQ was removed from cultures by rinsing twice in warm PBS ( 2 min each). Cultures were fixed with $4 \%$ paraformaldehyde $(10 \mathrm{~min})$ and briefly rinsed in three graded steps of ethanol (70, 80 and 90\%) and embedded in Fluoroshield (Molecular Probes). Polynucleated myotubes ( $500 \mathrm{n} M$ of Sytox nucleic acid stained $>5$ myonuclei of Sytox nucleic acid, Molecular Probes) exhibiting the red DAQ fluorescence (i.e. NO signals) were counted under epifluorescence illumination (Zeiss Axioplan, Germany). DAQ-loaded co-cultures were costained with Alexa Fluor 488 green fluorescent BGTx label to identify AChR clusters on DAQ-positive myotubes at sites of neurite contacts. For comparison, BGTx clusters were counted (i) in aneuronal cultures with culture medium only, or (ii) in myotube cultures incubated overnight with fresh conditioned medium from parallel p75-selected neuron cultures. The amount of green fluorescent BGTx-labeled AChR clusters superimposed on DAQ-positive red-fluorescent double-stained myotube segments $(100 \mu \mathrm{m})$ was calculated as an index for neurite contact formation in co-cultures. All fluorescence markers were monitored with a multilaser confocal scanning microscope (TCS SP-2, Leica Microsystems, Bensheim, Germany) under standardized conditions (8-bit, image size: $1024 \times 1024$, voxel size: $100 \times 100 \mu \mathrm{m}$ ). The red-fluorescent intracellular signals were analyzed using a scale for pixel intensity (0-255 arbitrary units) of rectangular regions of interest $\left(\mathrm{ROI}=5,000 \mu \mathrm{m}^{2}\right)$ and plotted as percent deviations from baseline.

Prior to the DAQ loading protocol, parallel co-cultures were pretreated for 4-6 days in vitro with $20 \mathrm{n} M$ of the NOS-1 and NOS-2 inhibitor AMT (Alexis) diluted in DMEM, for $1 \mathrm{~h}$ at $37^{\circ} \mathrm{C} / 5 \% \mathrm{CO}_{2}$ atmosphere. AMT was removed by three rinses in $37^{\circ} \mathrm{C}$ prewarmed DMEM followed by the DAQ loading protocol. In these cultures, fluorescent DAQ signals were neither detectable in aneural nor in neurally contacted myotubes. Thus, the DAQ label reflects real-time and de novo production of intracellular NO in viable myotubes [36]. DAQ-preloaded myotubes were also incubated with s-agrin 4,19 (50 $\mathrm{p} M$ in DMEM, gift of W. Hoch, MPI Tübingen, Germany) for $20 \mathrm{~h}$ at $37^{\circ} \mathrm{C}$ [23], or co-cultured with sensory DRG neurons according to previously described methods [30]. In the latter experiments, DAQ signals in fused myotubes were measured as described. Values represent means \pm SEM. The results were analyzed after variance analysis and Student's $t$ test at a significance level of $p<0.05$.

\section{Results}

Survival and neurite outgrowth of $\mathrm{p} 75$-selected spinal cord motoneurons were evident $48-72 \mathrm{~h}$ after seeding as verified by synaptotagmin-immunopositive neural somata and neuritic processes expanding over elongated myotubes (fig. 1A). Double immunolabeling showed a selective clustering of AChR on the myotube surface verified by postsynaptic marker $\alpha B G T x$ at sites of neurite contacts verified by the presynaptic immunomarker synaptotagmin (fig. 1B). At higher magnifications of cluster areas, sequential image analysis confirmed the close spatial relationship between outgrowing neurites and AChR aggregates on myotubes (fig. 1C, D). The yellow pixels generated by region-oriented segmentation image analysis reflect a 1:1 overlap of fluorescent-labeled structures of the neurite process and of $\mathrm{AChR}$ aggregates at sites of intimate contacts (fig. 1D).

The overall pattern and amount of AChR aggregates on myotubes from neuron-myotube co-cultures (fig. 2A) are very different from aneural myotubes (fig. 2B), and from those myotubes which were grown in the presence of p75 neuron-conditioned medium. The quantitative evaluation showed an increase in the number of specific AChR clusters (i.e. macroclusters) on defined myotube segments $(3.9 \pm 0.3$ clusters $/ 100-\mu \mathrm{m}$ segment, $\mathrm{n}=60)$ in co-cultures (fig. 2C) when compared to aneuronal cultures, i.e. myotubes cultured under identical conditions without neurons $(0.6 \pm 0.3$ clusters/segment, $\mathrm{n}=40)$. Addition of neuron-conditioned medium containing soluble factors released by neurons did not result in comparable membrane clustering (fig. 2C).

In order to investigate the time course of early neuron/ myotube interactions, the degree of specific BGTx cluster patterns on myotubes was counted. Coclustering was detected starting from day 5 after seeding (fig. 2D). A significant increase in the amount of BGTx-cluster-positive myotubes was however detected 5-6 days in vitro after seeding of p75 motoneurons reflecting onset of major triggering mechanisms supporting AChR membrane clustering. As neurites grew out in co-cultures as early as $48 \mathrm{~h}$ 
Fig. 1. Merged confocal images of neuronmyotube co-cultures (black/white reproduction). A $\alpha$ BGTx (red)-labeled AChR clusters (white spots) on a myotube (MT, thin arrow) induced by neurite contacts (arrow) originating from a p75-selected neuronal cell body (large arrow) immunolabeled with greenfluorescent (white) presynaptic marker antisynaptotagmin (SynTag) at 4 days in vitro. B Overview showing immunostained neurite (green, arrows) traversing BGTx clusters (red) on the myotube membrane. C Magnification of a neuron-myotube contact region (5-6 days in vitro). D Region-oriented segmentation imaging (ROSI) reflecting adjacent pixel analysis. The yellow pixels (white in $b / w)$ reflect $1: 1$ overlapping of pre- and postsynaptic structures (i.e. neurite process vs. AChR clusters). Bars $=20 \mu \mathrm{m}(\mathbf{A}, \mathbf{B})$ and $10 \mu \mathrm{m}(\mathbf{C}, \mathbf{D})$.
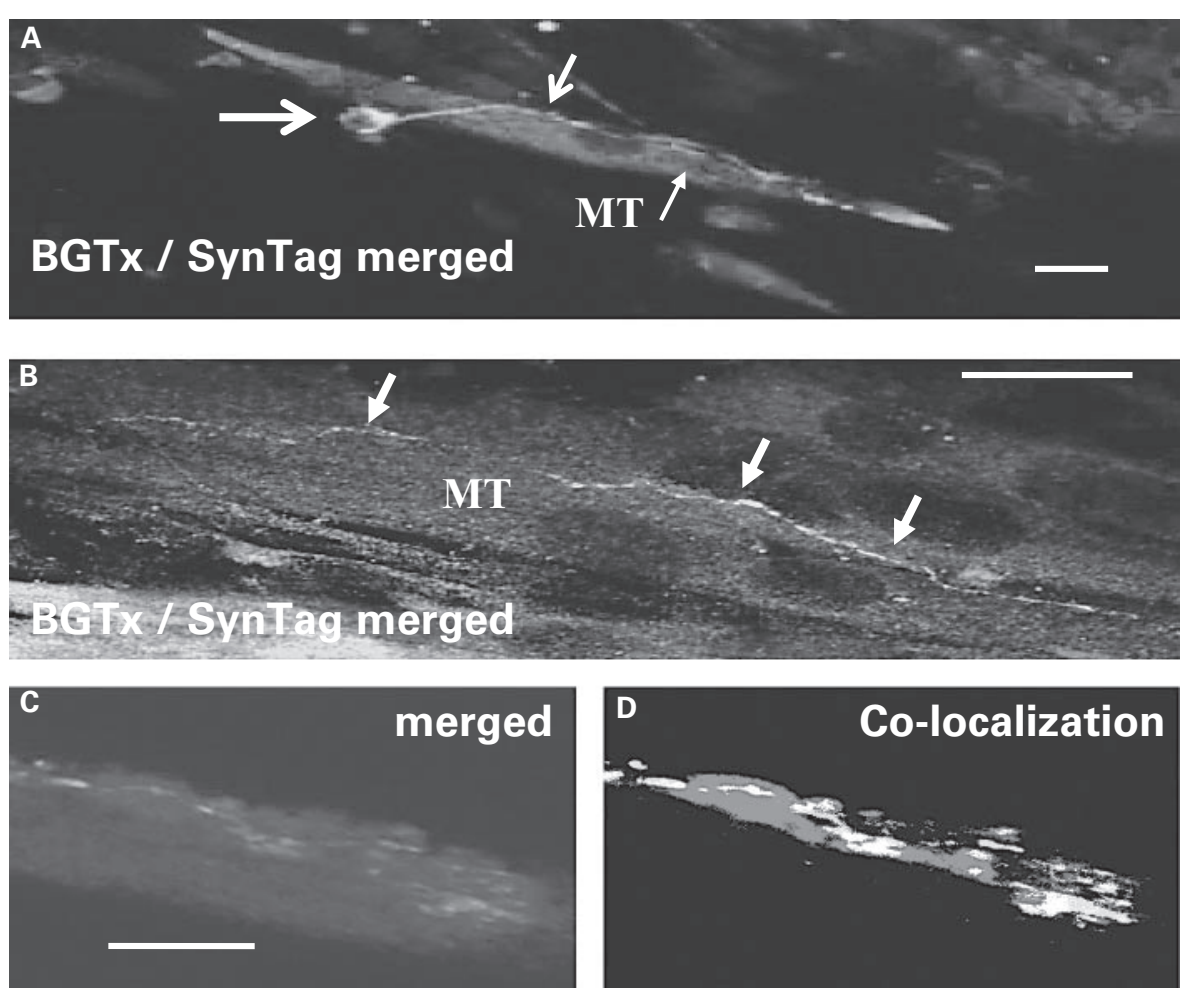

after seeding (anti-NF immunomarker), we concluded that neurite-myotube contacts must have established after 3-5 days in vitro. A decline in the BGTx clusters was noted in co-cultures at later developmental stages in vitro.

We next blocked NO biosynthesis in myotube cultures by treatment of fused myotubes with various concentrations of known NOS inhibitors followed by colorimetric NO assay (fig. 3). Blocking with variable final concentrations depending on NOS inhibitors decreased the concentration of NO in myotube cultures as compared to myotube-only cultures without blocking treatments $(n=6)$. In the absence of NOS blockers, NO levels were significantly elevated in fused myotubes and further increased in cocultures (fig. 3).

The formation of intracellular NO was directly visualized in individual myotubes by preloading them with the non-fluorescent NO-sensitive compound DAQ, which, in the presence of NO, generates a strong red fluorescence detected by confocal microscopy. Quantification was done by ROI pixel intensity measurements (scale: 0-255 arbitrary units). In myotubes pretreated with the NOS inhibitor AMT, DAQ red fluorescence (verifying intracellular NO) was not detectable in the myotube sarcosol
$(12.25 \pm 7)$ as determined by ROI-pixel intensity measurements, suggesting that the in vitro DAQ protocol specifically stained de novo formation of intracellular NO (fig. 4A). Without blocking, aneural myotubes revealed only weak DAQ red fluorescence $(38.9 \pm 9)$ thus reflecting intracellular basal levels of NO (fig. 4B). Robust DAQ red fluorescence (ROI pixel intensity $98.0 \pm 3$ ) was however detectable in many myotubes in co-culture thus reflecting an approximately $159 \%$ increase of the relative fluorescence at the ROIs measured compared to basal values found in other myotubes of the same co-cultures (fig. 4C). The DAQ fluorescence in responding myotubes was always diffusely distributed and, for example, not restricted to membrane aggregations possibly induced at sites of neurite-myotube contacts. Neither the addition of the AChR aggregating molecule s-agrin nor co-cultures with sensory DRG neurons showed comparable effects on myotube DAQ fluorescence (not shown).

Costaining experiments with anti-NF and DAQ verified the presence of neurite processes on strongly red fluorescent, i.e. DAQ-positive, myotubes only (fig. 4D-E, see also 4C). Neurite processes were not detectable on DAQnegative myotubes adjacent to DAQ-positive myotubes (fig. 4E, inset). During axonal outgrowth and guidance, 

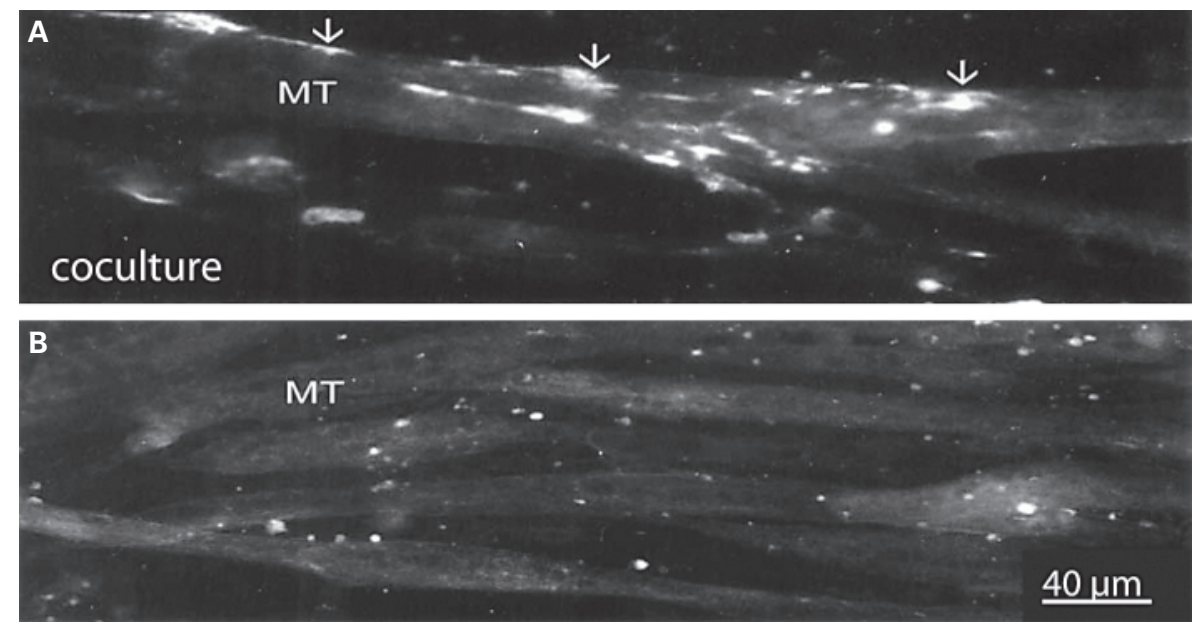

Fig. 2. Epifluorescence microscopy of $\alpha$ BGTx-labeled specific AChR 'macroclusters' (white spots) determined in co-cultures (A) versus aneural myotubes with few spontaneous 'microclusters' (B). C The number of BGTx clusters per myotube segment in coculture is significantly different from aneural cultures or from cultures supplemented with motoneuron-conditioned medium. D Graph shows percent changes in myotubes with BGTx-labeled AChR clusters versus clusterpositive aneural myotubes (set as $0 \%$ ) after 0-8 days in co-culture. Specific AChR clustering responses are lacking on myotubes at $1-4$ days in vitro (i.e. $<5 \%$ ) suggesting that physical contacts between neurites and myotubes were not yet established in co-culture. At 5-6 days in vitro, the number of specific BGTx-cluster-positive myotubes is significantly increased, thus monitoring neuronmyotube contacting in vitro. $\mathrm{Bar}=40 \mu \mathrm{m}$.

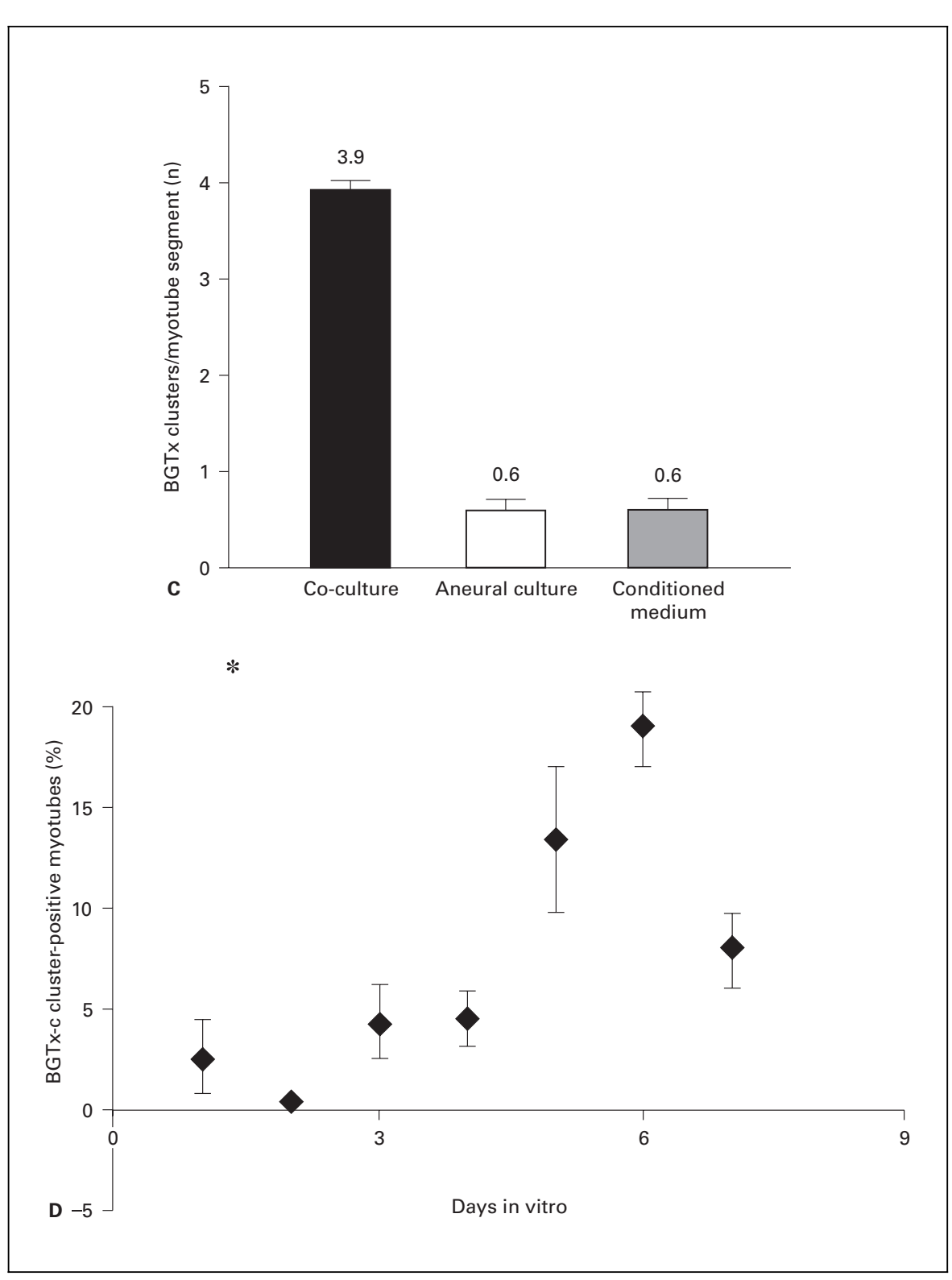


Fig. 3. Colorimetric NO assay of the culture medium after various treatments. Minimal amounts of NO were detected in myotube cultures following administration of various NOS inhibitor concentrations: $1 \mu M$ diphenyleiodonium chloride (DPI), $100 \mathrm{n} M$ 3-bromo-7-nitroindazole (3-B-7-NI), $20 \mathrm{n} M$ AMT or $1 \mu M$ n $\omega$-nitro- $L$-arginine (L-NNA) in culture medium (5 days in vitro). Elevated NO levels were detected in co-cultures $(41 \mu M)$ as compared to basal NO concentrations in myotube-only cultures $(20 \mu M)$ of the same developmental stage in vitro (5 days in vitro).

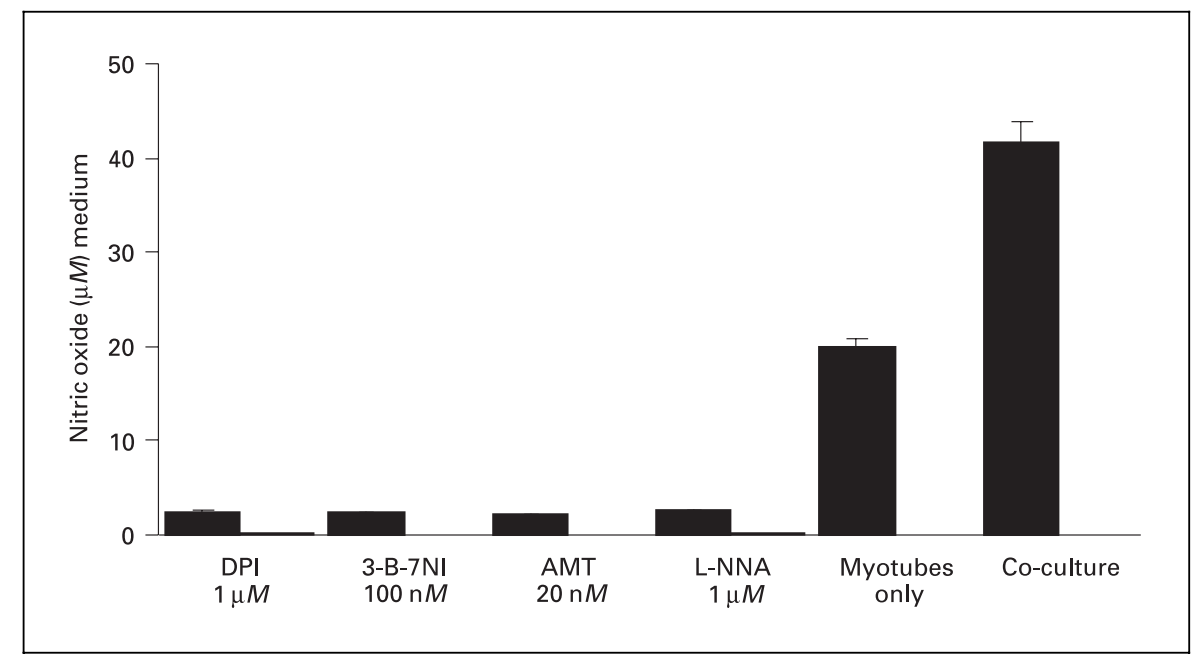

neurite extensions may have passed other myotubes before making contacts with their target myotubes in co-culture for 3-5 days in vitro (fig. 2D). Therefore, correlations between neurite immunostaining and DAQ fluorescence were not found.

We rather measured correlations between AChR aggregations (typical macroclusters induced by neurites) and DAQ fluorescence of target myotubes (fig. 5). We performed selective counts of fluorescent $\alpha B G T x$-labeled AChR clusters detectable in $100-\mu \mathrm{m}$ myotube segments from both DAQ-positive and DAQ-negative myotubes in co-culture and compared the results with DAQ-preloaded aneural cultures (fig. 5). Starting from 4 days in vitro onwards, the number of DAQ-positive myotubes expressing AChR macroclusters in co-culture was significantly increased by approximately 3 -fold compared to aneuronal myotube cultures. Intracellular NO production, therefore, becomes upregulated in myotubes upon neuromuscular contact as visualized by both strong sarcosolic DAQ red fluorescence in the presence of neurite-induced AChR aggregates detectable in co-cultures by confocal microscopy.

\section{Discussion}

The major findings of the present study were as follows: (i) p75 neurons isolated from embryonic chick spinal cords survived and extended neurites when co-cultured with $\mathrm{C} 2 \mathrm{C} 12$ myotubes, (ii) increased NO concentrations were determined in neuron-myotube co-cultures by colorimetric NO assay, (iii) myotubes in co-culture showed strong sarcosolic DAQ red-fluorescent stain when monitored by confocal laser microscopy, (iv) the DAQpositive myotubes in co-culture were always contacted by neurites identified by pre- and postsynaptic markers and (v) neither agrin nor sensory neurons produced similar effects on cultured myotubes. We suggest that endogenous NO formation in myotubes is dependent on neuronal signals resulting in NOS induction at the developing neuromuscular junction. The signaling cascades responsible for myotube NOS induction remain to be clarified.

To our knowledge, this is the first report on neuronal contact-induced NO formation in skeletal myotubes visualized by the NO-sensitive fluorescent marker DAQ by confocal microscopy. Cell markers like DAQ and DAF-2 (4,5-diaminofluorescein) are useful for specific and semiquantitative visualization of de novo formation of $\mathrm{NO}$ in viable cells in vitro and in vivo $[35,37,38]$. Induction of NO in myotubes was preferentially detectable in co-cultures with p75-selected neurons enriched from the motoneuron pool of the embryonic chicken lumbar spinal cord. Intimate neurite-myotube contacts were identified in coculture by the presence of postsynaptic AChR clusters on myotubes [39-43] and redistribution of ACh receptor and esterase mRNA [44-46].

Myotubes form postsynaptic myopodia (microprocesses) at sites of axonal contacts in Drosophila melanogaster embryos in vivo [47] and in neuron-myotube cocultures within the first $24 \mathrm{~h}$ in vitro [43] suggesting dynamic interactions following establishment of contacts between myotube and axon in vitro and in vivo. Initial events in neuromuscular synapse formation $[48,49]$ include agrin-induced aggregation of membrane complexes or macroclusters including the AChR, ion channel pro- 

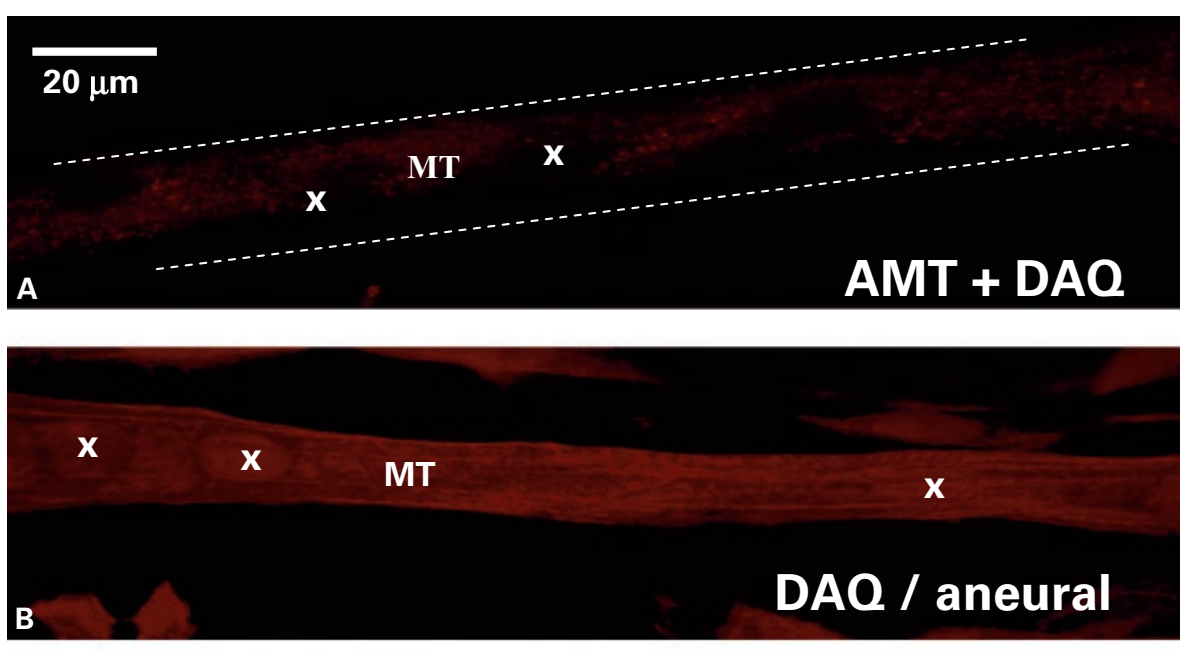

Fig. 4. Intracellular detection of $\mathrm{NO}$ in viable C2 myotubes preloaded with the NOsensitive compound DAQ. A Preincubation with the selective NOS inhibitor AMT resulted in negative DAQ fluorescence of myotubes (MT); the myonuclei (x) are spared. Broken lines denote the location of a DAQnegative myotube. B Aneural myotube cultures (5 days in vitro) show moderate DAQ fluorescence signals in sarcosol and myonuclei reflecting NO baseline production. C Co-cultures (4-5 days in vitro) with robust DAQ fluorescence signals (red) in myotubes reflecting elevated NO production; the myonuclei are masked by the large amounts of intracellular DAQ signals. D DAQ-positive myotube with a traversing anti- $\alpha$ NF-immunopositive neurite (green, vertical arrows) of an adjacent p75-selected neuronal soma (horizontal arrow). E DAQ-positive myotube with Cy2 $\alpha \mathrm{NF}$-immunostained neuritic process (arrows). DAQ-positive mononucleated myoblasts (x) located next to polynucleated myotubes (MT). Inset shows a neighboring myotube with weak DAQ fluorescence (broken lines) lacking NF-immunostained neurites (green) from the same coculture. A-E Bar $=20 \mu \mathrm{m}$ (inset, $40 \mu \mathrm{m}$ ).
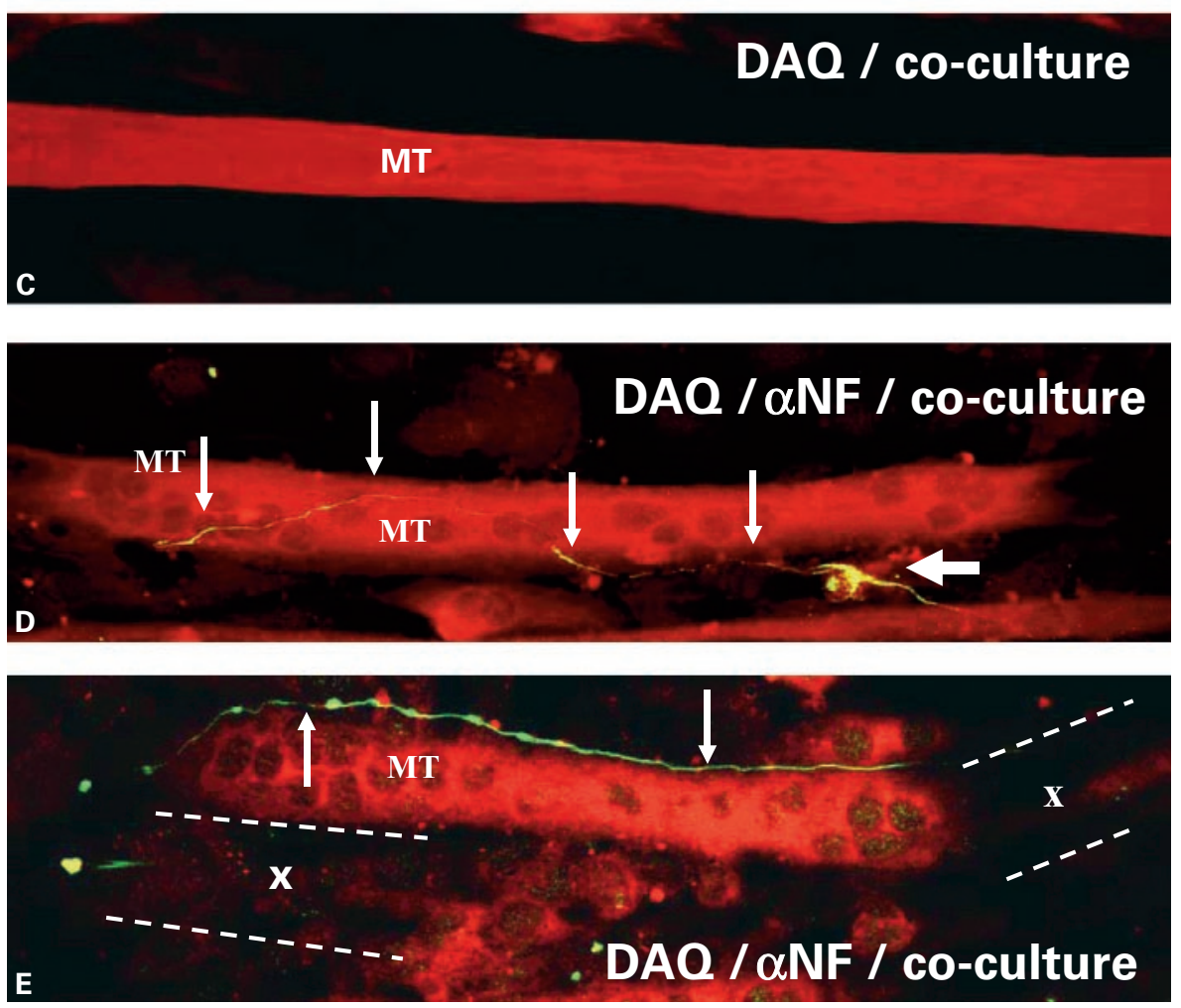

teins, or multiple adaptor proteins [50-53]. Agrin may act as one of several presynaptic signals for in vitro AChR clustering in cultured myotubes $[54,55]$. At the postsynaptic membrane, $\mathrm{NO}$ acts as a downstream mediator of the initial agrin-induced AChR clustering process [22, 56], supporting the notion that the agrin/NO-signaling pathway is likely involved in redistribution of NOS-1 from cytosol to synaptic membrane clusters previously proposed [23].

Neuromuscular Control of Nitric Oxide
The typical AChR macroclusters formed in vitro are known to be initiated by nerve-derived soluble agrin secretion at sites near nerve-muscle contacts [57, 58], whereas spontaneous clusters (i.e. microclusters) on myotubes may be induced by normal synthesis of muscle proteoglycans [59], laminins [60] or by muscle-derived agrin for example [61, 62]. In our experimental model, soluble agrin did not induce strong DAQ red fluorescence in myotubes. Addition of agrin to myotube cultures may not be a

Neurosignals 2005;14:85-95 


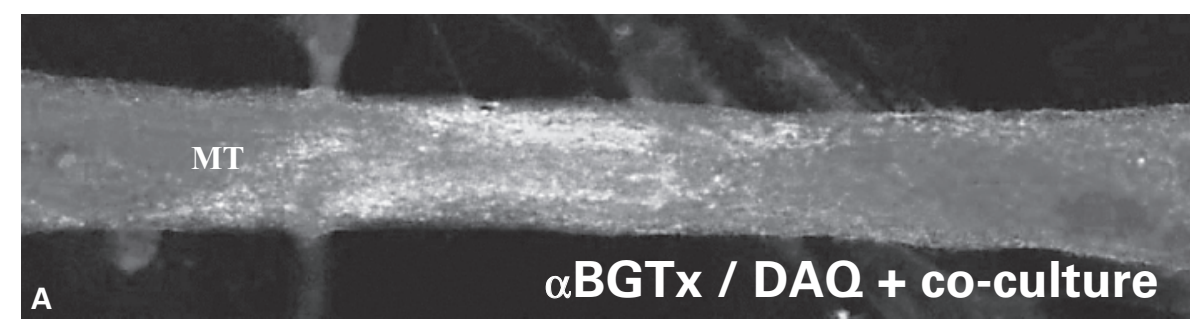

Fig. 5. A Merged confocal image of a DAQpositive (DAQ+) myotube (grey in b/w) with green fluorescent Alexa Fluor 488 conjugated BGTx macroclusters (white spots) in p75 neuron/myotube co-culture. B Quantification of neurite-induced AChR aggregates counted at DAQ-positive (DAQ+) vs. DAQnegative (DAQ-) myotubes. After 4-5 days in vitro, a significant increase in the relative amount of AChR macroclusters per DAQpositive myotube is detectable in co-culture, whereas DAQ-negative myotubes only revealed few spontaneous microclusters. Bar $=$ $10 \mu \mathrm{m}$.

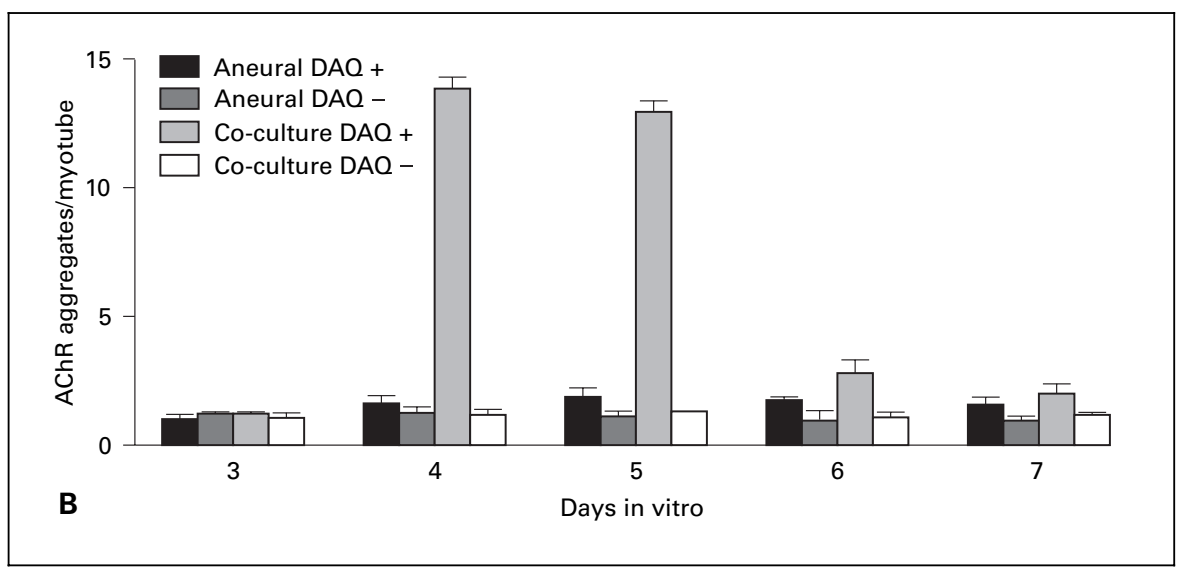

localized synaptic signal. Thus, lack of sarcosolic NO signals in agrin-treated myotubes may be due to more localized actions of agrin activity requiring parallel-operating presynaptic or postsynaptic signaling molecules from motor axons or target muscle cells for example. Myotubes with spontaneous $\mathrm{AChR}$ aggregates were also seen in our co-cultures, which, however, always lacked strong DAQ fluorescence and pre- and postsynaptic markers. The presence of diffusible trophic signals in the culture medium would hardly explain the cluster patterns of innervated DAQ-positive myotubes in co-culture since motoneuron-conditioned medium had no significant effects on single myotube cultures. The BGTx macroclusters induced by first-formed motor axon contacts on target myotubes on days 3-5 in vitro (i.e. agrin dependent) may be reversible at later in vitro development (i.e. becoming agrin-independent spontaneous microclusters), possibly due to the lack of appropriate molecular signaling or limited trophic support and thus incomplete local organization of the synaptic machinery, or simply by reversible neurite-contacting mechanisms at former target myotubes in our experimental co-culture system.

Synaptic actions by NO have previously been discussed in terms of retrograde muscle-to-nerve NO signaling [21]. NOS is concentrated in neuromuscular junctions of adult and developing rats, mice or humans [16-18].
Cyclic GMP-dependent protein kinase is coexpressed with NOS at the neuromuscular junction [19], and NOS-1 is either linked directly via PDZ-PDZ domains or indirectly via PSD-95 to the membrane N-methyl- $D$-aspartic acid receptor in the brain [63] supporting the notion that local $\mathrm{Ca}^{2+}$ influx could be the trigger for NOS activity at the synaptic multimolecular complex. More recently, the molecular association between synapsin and NOS has been characterized [64]. The presence of muscle-specific receptor tyrosine kinases, muscle-specific kinase (MuSK) [65], found in embryonic muscle and neuromuscular junctions provide possible molecular cues for transmembrane signaling processes and second messenger pathways initiated by motoneuron-muscle contacts.

As principally all major NOS isoforms were found in skeletal myoblasts, the question arises which of the NOS isoforms might be responsible for the robust and diffuse myotube NO signals detected in our co-cultures. Attempts to further characterize a 'developmental-type' isoform of NOS in fused $\mathrm{C} 2$ myotubes by NOS-isoform-specific antisense mRNA transfection experiments (NOS-1-NOS-3) failed to show clear correlations between NOS isoform expression and the known biological effects (i.e. fusion competence) observed in vitro [unpubl. observation]. Redundantly or residually expressed active NOS isoforms in early development may thus be responsible for critical 
NO levels as a prerequisite for myoblast fusion and differentiation [66]. On the other hand, NOS isoform activity and thus NO levels may be regulated later in development subserving more spatial actions of NO addressing molecular targets of intracellular compartments of more differentiated skeletal muscle. As the mature pattern of skeletal muscle innervation, for example, is achieved at peri- and postnatal stages [67], it seems likely that the known redistribution of the cytosolic NOS to sarcolemma sites previously reported $[25,68]$ partly reflects myofiber innervation patterning in postnatal skeletal muscle differentiation.

However, the functional consequences of neuriteinduced NO production in myotubes are manifold. In skeletal muscle, NO mediates excitation-contraction coupling, mitochondrial and glucose metabolism, and autoregulation of the microvascular network [13]. Freely diffusible NO reacts with several classes of proteins, soluble guanylyl cyclases, sarcoplasmic ATPases, respiratory chain complexes as well as radical oxygen species termed ROS $[69,70]$. NO inhibits or activates the sarcoplasmic reticulum ryanodine receptor in muscle thereby modulating intracellular calcium levels [71]. In adult muscle from NOS-3-deficient mice, NO production and contractile functions were not impaired suggesting that NOS-1 is the major isoform which mediates intracellular NO signaling in mature muscle fibers [72]. Electrical stimulation of adult skeletal muscle preparations induces NOS-1, thus activity-dependent mechanisms may act as physiological triggers for skeletal muscle NO formation [73]. During development, NO is thought to participate in activitydependent synaptic suppression in neuromuscular [27] or central synapses [74], in neuronal proliferation and survival in cultured neuronal cells [75] and in Xenopus brain
[76], supporting the idea that similar events are regulated by retrograde muscle-to-nerve NO signals from skeletal muscle targets [77] critical for the assembly and maintenance of the postsynaptic apparatus [78]. In synaptogenesis and neurite outgrowth, NO acts by itself or in concert with other growth factor peptides [79]. Muscle-fiberderived NO signals may act as intrinsic molecular mediators for postsynaptic AChR expression [80] or plasticity control in muscle differentiation and innervation patterning as recently proposed for $\mathrm{AChR}$ aggregations formed at prospective synaptic regions of muscle fibers [81]. In general, neurite-induced NO formation in vitro may be part of synergistic effects of muscle- and/or nerve-derived peptides, for example, acting on the cellular and molecular level at early or late synaptogenesis [82-84].

In conclusion, the present findings provide evidence for elevated NO levels in neuron-myotube co-cultures. De novo formation of endogenous $\mathrm{NO}$ has been directly visualized in viable target myotubes which were contacted by motor axons. Both agrin and sensory neurons failed to show comparable results in our model. Elucidation of the interactive pre- and postsynaptic molecular signals involved in NO biosynthesis during nerve-muscle interactions will help to further understand target-dependent NO signaling in development, differentiation and plasticity of the neuromuscular system.

\section{Acknowledgments}

This work was supported by grants from the 'Deutsche Forschungsgemeinschaft' (Bl 259/3-3) to D.B. Special thanks go to Gudrun Schiffl for expert technical support.

\section{References}

$>1$ Bredt DS, Snyder SH: Nitric oxide: A physiological messenger molecule. Annu Rev Biochem 1994;63:175-195.

$>2$ Bredt DS, Snyder SH: Isolation of nitric oxide synthetase, a calmodulin-requiring enzyme. Proc Natl Acad Sci USA 1990;87:682-685.

$>3$ O'Dell TJ, Huang PL, Dawson TM, Dinerman JL, Snyder SH, Kandel ER, Fishman MC: Blockade of LTP by inhibitors of nitric oxide synthase (NOS) in mice lacking neuronal NOS suggests a role for endothelial NOS. Science 1994;265:542-546.
-4 Ogilvie P, Schilling K, Billingsley ML, Schmidt $\mathrm{HH}$ : Induction and variants of neuronal nitric oxide synthase type-1 during synaptogenesis. FASEB J 1995;9:799-806.

$>5$ Stamler JS, Lamas S, Fang FC: Nitrosylation, the prototypic redox-based signaling mechanism. Cell 2001;106:675-683.

$>6$ Wang Y, Newton DC, Marsden PA: Neuronal NOS: Gene structure, mRNA diversity, and functional relevance. Crit Rev Neurobiol 1999; 13:21-43.

7 Bredt DS, Snyder SH: Nitric oxide as a neuronal messenger. Trends Pharmacol Sci 1991;12: 125-128.
-8 Blottner D: Nitric oxide and fibroblast growth factor in autonomic nervous system: Short- and long-term messengers in autonomic pathways and target-organ control. Progress Neurobiol 1997;51:423-438.

-9 Vogel M, Lück G, Bachmann S, Blottner D: NOS type-1 mRNA expression and protein localization in spinal autonomic neurons. Neuroreport 1997;8:3389-3393.

$>10$ Nakane M, Schmidt HH, Pollock JS, Förstermann U, Murad F: Cloned human brain nitric oxide synthase is highly expressed in skeletal muscle. FEBS Lett 1993;316:175-180. 
$\$ 11$ Balon TW, Nadler JL: Nitric oxide release is present from incubated skeletal muscle preparations. J Appl Physiol 1994;77:2519-2521.

12 Kobzik L, Reid MB, Bredt DS, Stamler JS: Nitric oxide in skeletal muscle. Nature 1994;372: 546-568.

13 Stamler JS, Meissner G: Physiology of nitric oxide in skeletal muscle. Physiol Rev 2001;81: 209-237.

14 Silvagno F, Xia H, Bredt DS: Neuronal nitric oxide synthase-mu, an alternatively spliced isoform expressed in differentiating skeletal muscle. J Biol Chem 1996;271:11204-11208.

15 Brenman JE, Chao DS, Gee SH, McGee AW, Craven SE, Santillano DR, Wu Z, Huang F, Xia H, Peter MF, Froehne SC, Bredt DS: Interaction of nitric oxide synthase with postsynaptic density protein PSD-95 and alpha1-syntrophin mediated by PDZ domains. Cell 1996;84: 757-767.

16 Kusner LL, Kaminski HJ: Nitric oxide synthase is concentrated at the skeletal muscle end plate. Brain Res 1996;730:238-242.

17 Olivier L, Coureau O, Courtois Y, Vigny M: Accumulation of NO-synthase (type-1) at the neuromuscular junction. Neuroreport 1996;22: 924-926.

-18 Yang CC, Alvarez RB, Engel WK, Haun CK, Askanas V: Immunolocalization of nitric oxide synthase at the postsynaptic domain of human and rat neuromuscular junctions - Light and electron microscopic studies. Exp Neurol 1997; 148:34-44.

-19 Chao DS, Silvagno F, Xia H, Cornwell TL, Lincoln TM, Bredt DS: Nitric oxide synthase and cyclic GMP-dependent protein kinase concentrated at the neuromuscular endplate. Neuroscience 1997;76:665-672.

20 Schoser BG, Behrends S: Soluble guanylyl cyclase is localized at the neuromuscular junction in human skeletal muscle. Neuroreport 2001; 12:979-981

21 Brenman JE, Bredt DS: Synaptic signaling by nitric oxide. Curr Opin Neurobiol 1997;7:374378.

22 Jones MA, Werle MJ: Nitric oxide is a downstream mediator of agrin-induced acetylcholine receptor aggregation. Mol Cell Neurosci 2000; 16:649-660.

-23 Lück G, Hoch W, Hopf C, Blottner D: Nitric oxide synthase (NOS 1) coclustered with agrininduced AChR-specializations on cultured skeletal myotubes. Mol Cell Neurosci 2000;16: 269-281.

24 Ebert JG, Zelenka M, Gath I, Gödtle-Armbrust U, Förstermann U: Colocalization but differential regulation of neuronal NO synthase and nicotinic acetylcholine receptor in $\mathrm{C} 2 \mathrm{C} 12$ myotubes. Am J Physiol Cell Physiol 2002;284: C1065-C1072.

- 25 Lück G, Oberbäumer L, Blottner D: In situ identification of neuronal nitric oxide synthase (NOS-I) mRNA in mouse and rat skeletal muscle. Neurosci Lett 1998;246:77-80.

26 Blottner D, Lück G: Just in time and place: NOS/NO system assembly in neuromuscular junction formation. Microsc Res Tech 2001; $55: 171-180$
27 Wang T, Xie Z, Lu B: Nitric oxide mediates activity-dependent synaptic suppression at developing neuromuscular synapses. Nature 1995;374:262-266

28 Thomas S, Robitaille R: Differential frequency-dependent regulation of transmitter release by endogenous nitric oxide at the amphibian neuromuscular synapse. J Neurosci 2001;21: 1087-1095.

29 Godfrey EW, Schwarte RC: The role of nitric oxide signaling in the formation of the neuromuscular junction. J Neurocytol 2003;32:591602 .

30 Blottner D, Unsicker K: Spatial and temporal patterns of neurotrophic activities in rat adrenal medulla and cortex. Dev Brain Res 1989; 48:243-253.

31 Camu W, Henderson CE: Purification of embryonic rat motoneurons by panning on a monoclonal antibody to the low-affinity NGF receptor. J Neurosci Methods 1992;44:59-70.

32 Juurlink BH, Munoz DG, Devon RM: Calcitonin-gene-related peptide identifies spinal motoneurons in vitro. J Neurosci Res 1990;26: 238-241.

33 Boucher JL, Moali C, Tenu JP: Nitric oxide biosynthesis, nitric oxide synthase inhibitors and arginase competition for $L$-arginine utilization. Cell Mol Life Sci 1999;55:1015-1028.

34 Bland-Ward PA, Moore PK: 7-Nitro-indazole derivates are potent inhibitors of brain, endothelium and inducible isoforms of nitric oxide synthase. Life Sci 1995;57:PL131-PL135.

35 Blottner D, Gerlach EM, Reicheneder C, Lück G: Neuronal regulation of nitric oxide synthase type-1 in motoneuron-myotube co-cultures. Soc Neurosci Abstr 2000;26:411.11.

36 Chen X, Sheng C, Zheng X: Direct nitric oxide imaging in cultured hippocampal neurons with diaminoanthraquinone and confocal microscopy. Cell Biol Int 2001;25:593-598.

-37 von Bohlen und Halbach O, Albrecht D, Heinemann U, Schuchmann S: Spatial nitric oxide imaging using 1,2, diaminoanthraquinone to investigate the involvement of nitric oxide in long-term potentiation in rat brain slices. Neuroimage 2002;15:633-639.

38 Lopez-Figueroa MO, Caamano C, Marin R, Guerra E, Alonso R, Morano MI, Akil H, Watson SJ: Characterization of basal nitric oxide production in living cells. Biochim Biophys Acta 2001;1540:253-264

-39 Anderson MJ, Cohen MW: Nerve-induced and spontaneous redistribution of acetylcholine receptors on cultured muscle cells. J Physiol 1977;268:757-773.

40 Cohen MW, Moody-Corbett F, Godfrey EW: Former neuritic pathways containing endogenous neural agrin have synaptogenic activity. Dev Biol 1995; 167:458-468.

41 Dutton EK, Uhm CS, Samuelsson SJ, Schaffner AE, Fitzgerald SC, Daniels MP: Acetylcholine receptor aggregation at nerve-muscle contacts in mammalian cultures: Induction by ventral spinal cord neurons is specific to axons. $\mathbf{J}$ Neurosci 1995;15:7401-7416.
42 Daniels MP, Lowe BT, Shah S, Ma J, Samuelsson SJ, Lugo B, Parakh T, Uhm CS: Rodent nerve-muscle cell culture system for studies of neuromuscular junction development, refinements and applications. Microsc ResTech 2000;49:26-37.

43 Uhm CS, Neuhuber B, Lowe B, Crocker V, Daniels MP: Synapse-forming axons and recombinant agrin induce microprocess formation on myotubes. J Neurosci 2001;21:96789689.

44 Bursztajn S, Berman SA, Gilbert W: Differential expression of acetylcholine receptor mRNA in nuclei of cultured muscle cells. Proc Natl Acad Sci USA 1989;86:2928-2932.

45 Choi RC, Pun S, Dong TT, Wan DC, Tsim KW: NG108-15 cells induce the expression of muscular acetylcholine esterase when co-cultured with myotubes. Neurosci Lett 1997;236: $167-170$

-46 Grubic Z, Komel R, Walker WF, Miranda AF Myoblast fusion and innervation with rat motor nerve alter distribution of acetylcholinesterase and its mRNA in cultures of human muscle. Neuron 1995;14:317-327.

47 Ritzenthaler S, Suzuki E, Chiba A: Postsynaptic filopodia in muscle cells interact with innervating motoneuron axons. Nat Neurosci 2000; 3:1012-1017

48 Burden SJ: The formation of the neuromuscular synapse. Genes Dev 1998;12:133-148.

49 Meier T, Wallace B: Formation of the neuromuscular junction, molecules and mechanisms. Bioessays 1998;20:819-829.

50 Campanelli JT, Hoch W, Rupp F, Kreiner T, Scheller RH: Agrin mediates cell contact-induced acetylcholine receptor clustering. Cell 1991;67:909-916

51 Colledge M, Froehner SC: Signals mediating ion channel clustering at the neuromuscular junction. Curr Opin Neurobiol 1998;8:357363

52 Ruegg MA, Bixby JL: Agrin orchestrates synaptic differentiation at the vertebrate neuromuscular junction. Trends Neurosci 1998;21:2227.

-53 Sanes JR, Lichtman JW: Development of the vertebrate neuromuscular junction. Annu Rev Neurosci 1999:22:389-442.

54 Cohen MW, Godfrey EW: Early appearance of and neuronal contribution to agrin-like molecules at embryonic frog nerve-muscle synapses formed in culture. J Neurosci 1992;12:29822992.

55 Campagna JA, Ruegg MA, Bixby JL: Evidence that agrin directly influences presynaptic differentiation at neuromuscular junctions in vitro. Eur J Neurosci 1997;9:2269-2283.

-56 Jones MA, Werle MJ: Agrin-induced AChR aggregate formation requires cGMP and aggregate maturation requires activation of cGMPdependent protein kinase. Mol Cell Neurosci 2004;25:195-204.

57 Reist NE, Werle MJ, McMahan UJ: Agrin released by motoneurons induces the aggregation of acetylcholine receptors at neuromuscular junction. Neuron 1999;28:865-868. 
58 Ma J, Lugo B, Shah S, Godfrey EW, Daniels MP: Synaptic localization and axonal targeting of agrin secreted by ventral spinal cord neurons in culture. J Neurobiol 2000;43:338-351.

$\checkmark 59$ Gordon H, Lupa M, Bowen D, Hall ZW: A muscle cell variant defective in glycosaminoglycan biosynthesis forms nerve-induced but not spontaneous clusters of the acetylcholine receptor and the $43 \mathrm{kDa}$ protein. J Neurosci 1993; 13:586-595.

60 Cohen MW, Jacobson C, Yurchenco PD, Morris GE, Carbonetto S: Laminin-induced clustering of dystroglycan on embryonic muscle cells: Comparison with agrin-induced clustering. J Cell Biol 1997;136:1047-1058.

-61 Lieth E, Cardarsis CA, Fallon JR: Musclederived agrin in cultured myotubes: Expression in the basal lamina and at induced acetylcholine receptor clusters. Dev Biol 1992;149:4154.

62 Burgess RW, Nguyen QT, Son YJ, Lichtman JW, Sanes JR: Alternatively spliced isoforms of nerve and muscle-derived agrin, their role at the neuromuscular junction. Neuron 1999;23: 33-44.

-63 Christopherson KS, Hillier BJ, Lim WA, Bredt DS: PSD-95 assembles a tenary complex with the N-methyl-D-aspartic acid receptor and a bivalent neuronal NO synthase PDZ domain. J Biol Chem 1999;274:27467-27473.

64 Jaffrey SR, Benfenati F, Snowman AM, Czernik AJ, Snyder SH: Neuronal nitric oxide localization mediated by a tenary complex with synapsin and CAPON. Proc Natl Acad Sci USA 2002;99:3199-3204.

65 Valenzuela DM, Stitt TN, DiStefano PS, Rojas E, Mattsson K, Compton DL, Nunez L, Park JS, Gies DR, Thomas S, Le Beau MM, Fernald NG, Copeland NG, Jenkins NA, Burden SJ, Glass DJ, Yancopoulos GD: Receptor tyrosin kinase specific for the skeletal muscle lineage: Expression in embryonic muscle, at the neuromuscular junction, and after injury. Neuron 1995; 15:573-584.
66 Lee KH, Baek MY, Moon KY, Song WK, Chung $\mathrm{CH}$, Ha DB, Kang MS: Nitric oxide as a messenger molecule for myoblast fusion. J Biol Chem 1994;269:14371-14374.

67 Jansen JK, Fladby T: The perinatal reorganization of the innervation of skeletal muscle in mammals. Prog Neurobiol 1990;34:39-90.

68 Blottner D, Lück G: Nitric oxide synthase (NOS) in mouse skeletal muscle development and differentiated myoblasts. Cell Tissue Res 1998;292:293-302.

69 Reid MB: Role of nitric oxide in skeletal muscle, synthesis, distribution and functional importance. Acta Physiol Scand 1998;162:401409.

70 Murrant CL, Reid MB: Detection of reactive oxygen and reactive nitrogen species in skeletal muscle. Microsc Res Tech 2001;55:236-248.

71 Hart JD, Dulhunty AF: Nitric oxide activates or inhibits skeletal muscle ryanodine receptors depending on its concentration, membrane potential and ligand binding. J Membr Biol 2000; 173:227-236.

72 Hirschfeld W, Moody MR, O'Brien WE, Gregg AR, Bryan RM, Reid MB: Nitric oxide release and contractile properties of skeletal muscle from mice deficient in type III NOS. Am J Physiol 2000;278:R95-R100.

73 Reiser PJ, Kline WO, Vaghy PL: Induction of neuronal nitric oxide synthase in skeletal muscle by chronic electrical stimulation. J Appl Physiol 1997;82:1250-1255.

74 Schuman EM, Madison DV: Nitric oxide and synaptic function. Annu Rev Neurosci 1994; 17:153-183.
5 Oppenheim RW, Wiese S, Prevette D, Armanini M, Wang S, Houenou LJ, Holtmann B, Gotz R, Peunova N, Enikolopov G: Nitric oxide triggers a switch to growth arrest during differentiation of neuronal cells. Nature 1995; 375:68-73.

76 Peunova N, Scheinker V, Cline H, Enikolopov $\mathrm{G}$ : Nitric oxide is an essential negative regulator of cell proliferation in Xenopus brain. $\mathrm{J}$ Neurosci 2001;21:8809-8818.

$>77$ Connor EA, Smith MA: Retrograde signaling in the formation of the neuromuscular junction. J Neurobiol 1994;25:722-739.

78 Sanes JR, Lichtman JW: Induction, assembly, maturation and maintenance of a postsynaptic apparatus. Nat Rev Neurosci 2001;2:791-805.

79 Hindley S, Juurlink BH, Gysbers JW, Middlemiss PJ, Herman MA, Rathbone MP: Nitric oxide donors enhance neurotrophin-induced neurite outgrowth through a cGMP-dependent mechanism. J Neurosci Res 1997;47:427-439.

80 Shiao T, Fond A, Deng B, Wehling-Henriks M, Adams ME, Froehner SC, Tidball JG: Defects in neuromuscular junction structure in dystrophic muscle are corrected by expression of a NOS transgene in dystrophin-deficient muscles, but not in muscles lacking $\alpha$ - and $\beta_{1}$-syntrophins. Hum Mol Genet 2004;13:18731884.

81 Arber S, Burden SJ, Harris A: Patterning of skeletal muscle. Curr Opin Neurobiol 2002;12: 100-103.

82 Snider WD, Lichtman JW: Are neurotrophins synaptotrophins? Mol Cell Neurosci 1996;7: 433-442.

$>83$ Lin W, Burgess RW, Dominguez B, Pfaff SL, Sanes JR, Lee KF: Distinct roles of nerve and muscle in postsynaptic differentiation of the neuromuscular synapse. Nature 2001;410: 1057-1064.

84 Poo MM: Neurotrophins as synaptic modulators. Nat Rev 2001;2:24-32. 